\title{
PROJECTION TECHNIQUES FOR SEPARATION OF MULTIPLE SECONDARY SURVEILLANCE RADAR SOURCES IN A REAL ENVIRONMENT
}

\section{N. PETROCHILOS}

\author{
CReSTIC, University of Reims \\ Moulins de la Housse, BP 1039 \\ 51687 Reims Cedex 2, France \\ email: petro@ieee.org
}

G. GALATI, E. PIRACCI

\begin{abstract}
Secondary Surveillance Radar (SSR) based on multilateration principle and omni-directional antennae are operational today $[1,2]$. We proposed new algorithms to separate a mixture of overlapping SSR replies on a $M$-elements antenna in previous works $[3,4]$, other solutions were also proposed in the literature $[5,6,7]$. Unfortunately, all have either some shortcomings, or an expensive computational cost, or no simple practical implementation. Therefore, there is a need for reliable, simple, effective algorithms to separate multiple SSR signals. Real recorded signals in a live environment are used to demonstrate the effectiveness of the proposed techniques.
\end{abstract}

\section{INTRODUCTION}

Originally denominated "Identification Friend or Foe" (IFF) during the Second World War, the Secondary Surveillance Radar (SSR) operates on an interrogation-reply basis (remind that primary radars are based on echo-location). The radar emits an interrogation, eliciting from the airplanes in the illuminating beam a reply generated by an on-board SSR transponder, and emitted by an omni-directional antenna. The interrogation and the reply are pulse-position modulated finite-length signals at carrier frequency of 1030 and $1090 \mathrm{MHz}$ [8]. Two operational protocols currently coexist: previously un-addressed mode $\mathrm{A} / \mathrm{C}$ and newer mode $\mathrm{S}$, in which the ground station selectively address the aircrafts and permit short data communications between the ground interrogating station and the aircraft [9]. This new standard is intended to reduce the reply rate, and will ultimately replace the mode A/C. In [1], it was first proposed to use a distributed network of receive-only stations, which allow multi-lateration and enhance message detection, see Fig. 1.

However, with distributed systems there is a dramatic increase of received replies per unit time, causing overlapping between replies in some operating conditions such as the acquisition of new incoming signals or unsolicited

\author{
DISP and V. VOLTERRA CENTER, \\ Tor Vergata University \\ Via del Politecnico, 1 - 00133 Roma, Italy \\ email: g.galati@ieee.org
}

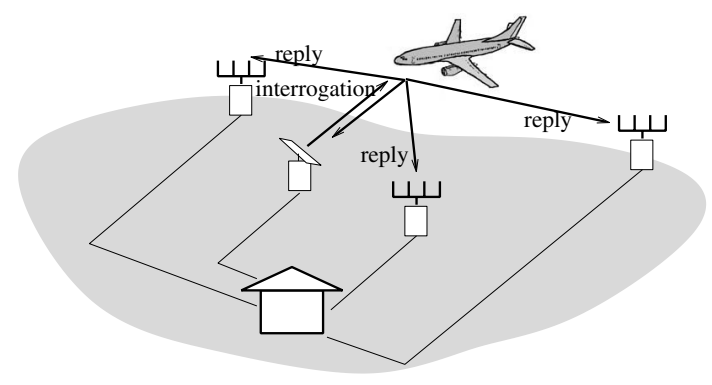

Fig. 1. The distributed SSR system (From [3]).

replies called "squitters". When replies overlap, very often the message transmitted by the aircraft is corrupted and cannot be recovered by conventional decoder (SIR-S), nor the aircraft can be located and identified.

Source separation based on array response matrix (for instance [6]) is not reliable, as it needs a perfect calibration and does not tolerate multipath. High-Order Statistic Separation algorithms [5] can't be used, as the replies are "pseudo-Gaussian" up to order $5[10,11]$. Deterministic separation algorithms have been considered in [3, 7], but they separate only mixtures of Mode S, they have a high computational burden, and need a joint diagonalisation of several tensors of order at least 3 . These deterministic algorithms have been designed for mode $\mathrm{S}$, and their pulse lengths are incommensurate with mode $\mathrm{A} / \mathrm{C}$ pulse lengths, so they fails in presence of Mode A/C. In this article, we present the extension of $[4,10]$, where the separation of two partially overlapping modes $\mathrm{S}$ uses the antenna diversity and projections. Our extension allows the separation of any kind of mixture of Mode A/C/S under mild conditions and work on a principle of deflation by projection. We demonstrate its effectiveness on a extensive library of real data acquired by an experimental platform that we designed in TU Delft.

\section{DATA MODEL}

We consider the reception of $d$ independent source signals on an $m$-element antenna array (of arbitrary form). 
The baseband antenna signals are sampled at frequency $T$ greater than the signal bandwidth and stacked in vectors $\mathbf{x}[n]$ (size $m$ ). After collecting $N$ samples, the observation model is

$$
\mathbf{X}=\mathbf{M} \cdot \mathbf{S}+\mathbf{N}
$$

where $\mathbf{X}=[\mathbf{x}[1], \cdots, \mathbf{x}[N]]$ is the $m \times N$ received signal matrix. $\mathbf{S}=[\mathbf{s}[1], \cdots, \mathbf{s}[N]]$ is the $d \times N$ source matrix, where $\mathbf{s}[n]=\left[s_{1}[n], \cdots, s_{d}[n]\right]^{T}$ is a stacking of the $d$ source signals (superscript $T$ denotes transpose). $\mathbf{N}$ is the $m \times N$ noise matrix, whose elements are temporally and spatially white. $\mathbf{M}$ is the $m \times d$ mixing matrix that contains the array signatures and the complex gains of the sources. We assume that the replies are independent (so uncorrelated), i.e. $\mathrm{E}\left\{s_{i} s_{j}^{*}\right\}=0$ for $i \neq j$, and $\mathbf{M}$ is full column rank $(d \leq m)$.

It is important to note that the sources are either mode $\mathrm{A} / \mathrm{C}$ or mode $\mathrm{S}$, so are packet-wise of different lengths, resp. $21.7 \mu$ s and $64 / 120 \mu \mathrm{s}$. The source consists of a binary sequence with alphabet $\{0,1\}$, modulated by a complex exponential due to a residual carrier frequency. Figure 2 present a typical case of mixed replies, where actually two mode $\mathrm{S}$ (in boxes) and one mode $\mathrm{A} / \mathrm{C}$ (not visible) are present.

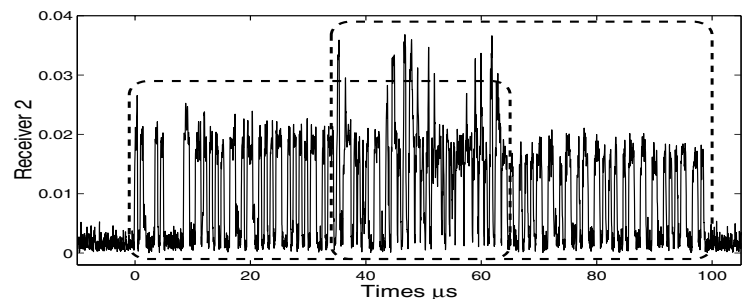

Fig. 2. A record of overlapped replies (case W5).

\section{THE ALGORITHM}

First we remind the algorithm proposed in [4] for the sake of comprehension, then its extension is presented.

\subsection{Projection Algorithm}

In [3], the MDA algorithm fails for partly overlapping mode $\mathrm{S}$ (with large time delay), moreover measurements of recorded signals have shown that a mixture of more than two mode $\mathrm{S}$ were still a rare event. Therefore in [4], we considered how to un-mix two mode $S$ having significantly different times of arrival, $t_{1}$ and $t_{2}$ (as it seems to be the case in Figure 2).

The algorithm begins with the detection of the $t_{i}$ 's. The data is sliced by times slots of 200 samples $(4 \mu \mathrm{s})$, on each time slot is performed a whiteness test [12] based on the Singular Value Decomposition, SVD [13], see Figure 3. This allows us to estimate the number of sources as a function of time, and to isolate the two time supports where each source is single.

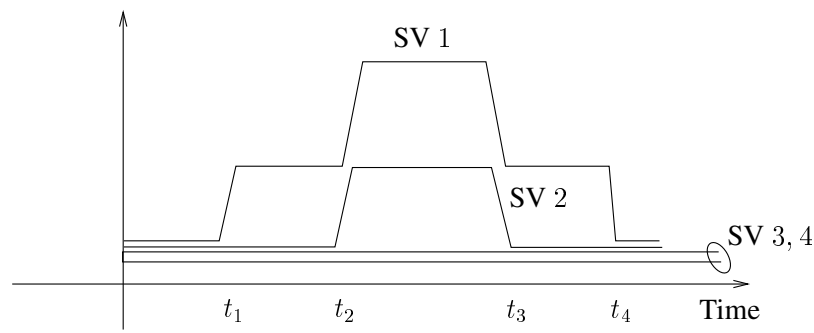

Fig. 3. The singular values as a function of time.

Be the notation (. $)^{(1)}$ the matrix collecting the subset of the columns related to the times $t_{1}$ till $t_{2}$ (selection of the columns). Similarly, we define the notation $(.)^{(2)}$ for the subset of the columns related to the times $t_{3}$ till $t_{4}$. Then we have the following relation:

$$
\begin{aligned}
& \mathbf{X}^{(1)}=\mathbf{M} \cdot \mathbf{S}^{(1)}+\mathbf{N}^{(1)} \\
& \mathbf{X}^{(2)}=\mathbf{M} \cdot \mathbf{S}^{(2)}+\mathbf{N}^{(2)}
\end{aligned}
$$

where the matrix $\mathbf{S}^{(1)}$ is the sub-matrix of $\mathbf{S}$ containing the samples in the time interval $\left[t_{1}, t_{2}\right]$. Therefore $\mathbf{X}^{(1)}$ contains only the first source and can be simplified as (resp. for $\left.\mathbf{X}^{(2)}\right)$ :

$$
\begin{aligned}
& \mathbf{X}^{(1)}=\mathbf{m}_{1} \cdot \mathbf{s}_{1}^{(1)}+\mathbf{N}^{(1)} \\
& \mathbf{X}^{(2)}=\mathbf{m}_{2} \cdot \mathbf{s}_{2}^{(2)}+\mathbf{N}^{(2)}
\end{aligned}
$$

where the $\mathbf{m}_{i}$ 's are the columns of $\mathbf{M}$, and the $\mathbf{s}_{i}$ 's the rows of $\mathbf{S}$.

Note that $\mathbf{X}^{(1)}$ and $\mathbf{X}^{(2)}$ are rank-one matrices in the noiseless case. By a SVD on $\mathbf{X}_{i}$, we can estimate the main vector $\hat{\mathbf{m}}_{i}$, which is the vector corresponding to the highest singular value.

Once the space signatures $\hat{\mathbf{m}}_{1}$ and $\hat{\mathbf{m}}_{2}$ have been identified, the matrix $\widehat{\mathbf{M}}$ is thus estimated. We finally multiply $\mathbf{X}$ by the Moore-Penrose pseudo-inverse of $\widehat{\mathbf{M}}\left(\widehat{\mathbf{M}}^{\dagger}=\right.$ $\left.\left(\widehat{\mathbf{M}}^{H} \widehat{\mathbf{M}}\right)^{-1} \widehat{\mathbf{M}}^{H}\right)$, and recover the estimated sources: $\widehat{\mathbf{S}}=$ $\widehat{\mathbf{M}}^{\dagger} \mathbf{X}$.

\subsection{Extension}

We consider now a mixture of several replies encoded in any mode. Assuming that $d \leq m$, we reduce the received signal dimension $m$ to $d$ by projecting $\mathbf{X}$ onto the signal subspace $\mathbf{V}_{d}$ (obtained by a SVD), such that:

$$
\mathbf{V}_{d}=\hat{\mathbf{M S}}+\hat{\mathbf{N}}
$$

with $\hat{\mathbf{M}}$ a reduced $d \times d$ square mixing matrix. From now on, we drop the hat from the notation. 
Our algorithm takes advantage of the packet form of the data to remove from one source after the other using the next proposition.

Proposition 1 Given d overlapping signals received by an d-element array with $N$ time samples, with the noiseless model: $\mathbf{V}_{d}=\mathbf{M S}$. Assuming that (A1) $\mathbf{M}$ is a $d \times d$ full rank matrix (and practically having a good conditioning number), (A2) one source of the mixture is present alone over a substantial time slot $\Delta T$ such that it is possible to detect it, (A3) this source is orthogonal to the other sources, then:

a) under (A1-2) a signal subspace of dimension d - 1 can be extracted, which contains only the $d-1$ remaining signals. $\beta$ ) adding (A3) permits to recover the one source.

Proof: We describe the algorithm as a proof. Using (A2), by a rank-one decomposition, we estimate the signature vector $\mathbf{m}_{1}$ of the one reply present over $\Delta T$.

Be the matrix $\mathbf{P}$ being a basis for the vector space $\mathbb{C}^{d}$, where we choose for the first vector $\frac{\mathbf{m}_{1}}{\left\|\mathbf{m}_{1}\right\|}(\|$.$\| stands for the Eu-$ clidean norm of a vector):

$$
\mathbf{P}=\left[\begin{array}{lllll}
\frac{\mathbf{m}_{1}}{\left\|\mathbf{m}_{1}\right\|} & \mathbf{w}_{2} & \mathbf{w}_{3} & \ldots & \mathbf{w}_{d}
\end{array}\right]
$$

where by construction, $\forall i \in\{2, d\} \mathbf{w}_{i}^{H} \mathbf{m}_{1}=0$.

Denotes $\mathbf{P}_{d-1}$ the sub-matrix containing only the last $d-1$ columns of $\mathbf{P}$. Then the resulting product $\mathbf{V}_{d-1}=$ $\mathbf{P}_{d-1}^{H} \mathbf{V}_{d}$ is a $(d-1) \times N$ matrix that contains only the sources 2 till $d$, such as

$$
\mathbf{V}_{d-1}=\mathbf{P}_{d-1}^{H} \mathbf{M}_{d} \mathbf{S}=\mathbf{P}_{d-1}^{H} \mathbf{M}_{d-1} \mathbf{S}_{d-1}
$$

where $\mathbf{S}_{d-1}$ are the last $d-1$ rows of $\mathbf{S}$, and $\mathbf{M}_{d-1}$ the last $d-1$ columns of $\mathbf{M}$.

Next, we need to prove that $\mathbf{P}_{d-1}^{H} \mathbf{M}_{d-1}$ is full rank square matrix. $\mathbf{P}$ and $\mathbf{M}$ are full rank square matrices, so the determinant of their product is non-zero. After a few derivation, it appears that the determinant is equal to

$$
\left|\mathbf{P}^{H} \mathbf{M}\right|=|| \mathbf{m}_{1}|| \cdot\left|\mathbf{P}_{d-1}^{H} \mathbf{M}_{d-1}\right|
$$

as the first determinant and the norm of the vector $\mathbf{m}_{1}$ are non-zero, it implies that the determinant, $\left|\mathbf{P}_{d-1}^{H} \mathbf{M}_{d-1}\right|$ is non-zero as well, and therefore the matrix is full rank, and point $\alpha$ ) is demonstrated.

Point $\beta$ ): as $\mathbf{m}_{1}$ is non-zero there is a $i$ such that the $i$-th component is $\left\{\mathbf{m}_{1}\right\}_{i} \neq 0$. Then the vectors set: $\left\{\mathbf{V}_{d-1}^{T} \mathbf{v}_{i}^{T}\right\}$ is full rank, performing a Gram-Schmidt orthogonalization on these vectors, the output of the last vector will be cleaned out of the contribution of $\mathbf{s}_{2}$ till $\mathbf{s}_{d}$, and therefore be proportional to $\mathbf{s}_{1}$. Practically, a reduced $\mathrm{QR}$ factorization on the matrix $\left[\mathbf{V}_{d-1}^{T} \mathbf{v}_{i}^{T}\right]$ delivers $\mathbf{s}_{1}^{T}$.
The Extended Projection Algorithm, EPA, is based on a deflation concept. Assuming that the sources are all orthogonal two by two, the recursive use of Proposition 1 allows us to separate the replies.

Beforehand, we estimate the noise standard deviation using a time interval which is chosen if 1 ) there is no crosscorrelation between channels (e.g. $\mathrm{E}\left\{x_{i}[n] x_{j}[n]\right\}=\delta_{i j}$ ), 2 ) if each channel output has a zero skewness and kurtosis, and 3 ) if the time auto-correlation of the channels outputs are approximately a Dirac function of time: $C_{i}(\tau)=$ $\mathrm{E}\left\{x_{i}[n] x_{i}^{*}[n+\tau]\right\}=\delta(\tau)$. The algorithm follows the next step:

1. The data is sliced in chunk of 400 samples $(8 \mu \mathrm{s})$ on which a SVD is performed (as in Figures 3 and 4).

2. For each slices, we use a whiteness test to decide if there are 0,1 , or more sources.

(a) if there exists at least one slice with one source, the slice with the largest estimated power is chosen to apply the Proposition 1. Go to step 3.

(b) if there only exist slices with either zero or more than one source, then we choose among the slices with several sources, the one which has the largest difference between the first and the second singular value. This choice is done hoping that we could estimate the signature vector of the main source. Go to step 3 .

(c) if no source are detected, the algorithm stops.

3. The extracted source ( $\beta$ ) from Prop. 1) is kept aside, while the estimated residual subspace $(\alpha)$ from Prop. 1 ) is used to feed the step 1.

Downstream, our separation algorithm, the extracted replies have to be decoded. To this end, we use a conventional decoder. One advantage is that under some conditions, a conventional decoder may decode two modes $\mathrm{A} / \mathrm{C}$ received on a channel. So if the separation fails, it gives an extra opportunity to decode the replies in given cases.

Remark: Note that this type of algorithm is not limited to SSR sources, but to any kind of packet-like sources.

\subsection{Conventional receiver}

Because we consider a practical algorithm, in the next section, we compare our algorithm over real data with an actual SSR system. Such system is composed of a LVA (Large Vertical Array) rotating antenna, conventional receiver and a plot extractor. The sum channel from the antenna supplies the signal to be processed by the conventional receiver, which consists of two steps: timing synchronization and pulse detection. For Mode A/C replies, the time synchronization is done by bracket detection, and for Mode S by 
preamble detection. Next to it, the pulse detection is performed using the estimated time reference and the signal amplitude estimate. The plot extractor provides to create and the refresh the airmobile tracks and shows to the ATC operator the scenario on a monitor.

\section{RESULTS}

The analysis is done using SSR signals received and recorded by means of an ad-hoc system implemented and owned by the Technical University of Delft (CAS/ IRCTR). This system is made up by a four-elements receive array connected to a wide band digital acquisition system ${ }^{1}$; thanks to it, a large amount of signals has been recorded during the summer 2003 [14].

\subsection{An example}

We first consider an example, the record W5, which has the worst behavior from the set of cases that has been presented in [15], where only pairs of mode $\mathrm{S}$ were investigated.

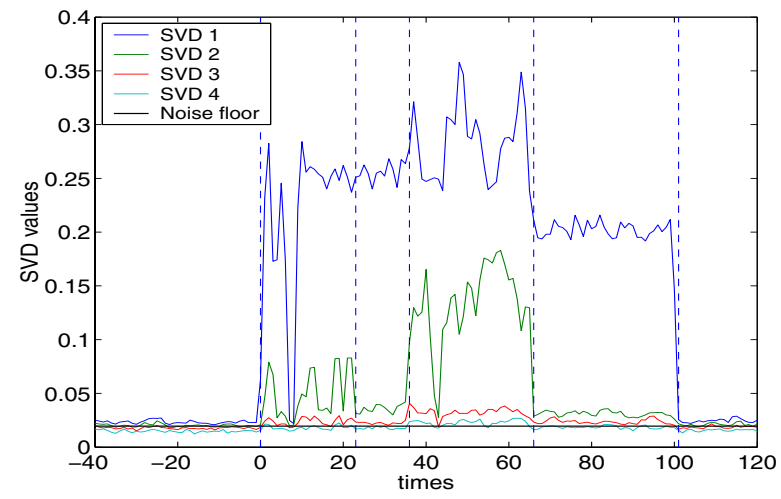

Fig. 4. Detection test over case W5 (time in $\mu$ s).

The SVD values used for the detection test are presented in Figure 4. Note that we detect an hidden Mode A/C that coincide exactly, by chance, with the preamble of the first Mode $\mathrm{S}$ reply.

Figure 5 presents the estimated replies by the extended algorithm from Proposition 1. We note that the separation is efficient. Remind that state of the art Mode S stations decoder can process only the leading mode $\mathrm{S}$ reply, and that other separation algorithms would be very expensive [5], or partly fail $[3,4,6,7]$.

\subsection{Performance analysis}

Next, we consider the data measured during the summer 2003. The campaign measurement lasted a week, and produced 131 files that have 130000 samples, so $2.6 \mathrm{~ms}$ long. The data was recorded for later off-line processing, so the

\footnotetext{
${ }^{1}$ http://cas.et.tudelft.nl/ nicolas/public_exp/
}
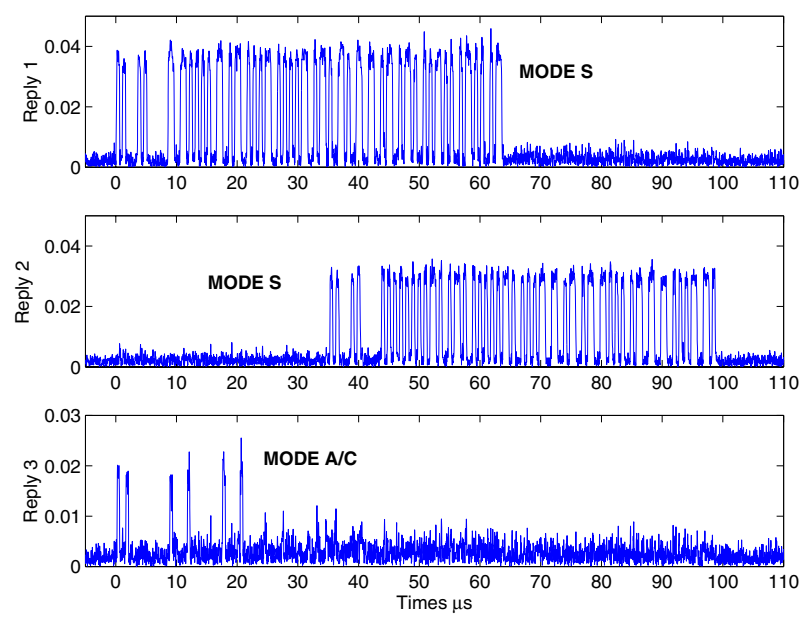

Fig. 5. Case w5: separation achieved by the Proposition 1.

quality of the measurement was not checked directly. We selected the most satisfying records, e.g. from the 3 best days, which represent then 70 records.

As the SSR replies are packet-wise, a record is made of several time intervals where a mixture of replies (or a single one) are present, and in-between only noise is present. We cut each record in several cases to keep only the times with replies; such that we obtain 651 different real cases to study.

\begin{tabular}{|l|c|c|c|c|c|}
\hline Case & $n_{i}$ & $n_{i} / N$ & C.R. & S. & S.+C.R. \\
\hline \hline Global & 651 & 1 & 0.46 & 0.88 & 0.61 \\
\hline \hline Single reply & 303 & 0.47 & 0.93 & 1.00 & 0.94 \\
\hline 2 replies & 190 & 0.29 & 0.07 & 0.90 & 0.39 \\
\hline 2 replies, R.D. & 38 & 0.06 & 0.00 & 0.00 & 0.11 \\
\hline 3 replies, & 84 & 0.13 & 0.00 & 0.93 & 0.29 \\
\hline 3 replies, R.D. & 15 & 0.02 & 0.00 & 0.33 & 0.20 \\
\hline 4 replies & 12 & 0.02 & 0.00 & 0.92 & 0.25 \\
\hline 4 replies, R.D. & 1 & 0.00 & 0.00 & 0.00 & 0.00 \\
\hline More replies & 8 & 0.01 & 0.00 & 0.63 & 0.00 \\
\hline \hline 2 $\rightarrow$ 8 replies & 348 & 0.53 & 0.04 & 0.78 & 0.31 \\
\hline
\end{tabular}

Table 1. Success table: Probability of event, success rate for the Conventional Receiver (C.R.), success rate for the Extended Projection Algorithm (S.), and for the Extended Projection Algorithm followed by the use of a conventional Receiver (S.+C.R.), for each number of replies. R.D. means "Rank Deficient" matrix M.

We present in table 1 the success rate of our algorithm compared to a Conventional Receiver. We classify the various cases by the number of present replies, independently if there are mode $\mathrm{A} / \mathrm{C}$ or mode $\mathrm{S}$. We also notify if the mixture of the sources has a mixing matrix $\mathbf{M}$ is rank deficient (for instance two replies impinge from the same direction), by denoting Rank Deficient (R.D.) in the table 1. The table presents the number of cases for each category, its percent- 
age, then the rate of success for the Conventionnal Receiver (CR), for the Extended Projection Algorithm (EPA), and for the EPA followed by the CR. A case is a success if all replies are detected and decoded.

We note that the number of 4 antennae was a good design choice as 4 or more replies occurs for only $2 \%$, having 4 antennae allows to perform noise reduction. First, we note that the EPA has an average success rate of only 0.88 . This is due to the fact that in some cases, it was not possible to find a slice of the data containing only one source. The other reason is that the mixing matrix $\mathbf{M}$ is rank deficient or that its conditioning number is high (i.e. (A1) in Prop. 1 is not respected). We note also that for 3 replies with Rank Deficiency, surprisingly, there are still some success. In these cases, after separation, there are two output channels, on the first output channel, there is one source, and on the second channel there are the two other replies, but separated in time. A similar effect occurs to the last category.

Considering the CR success rate for a single reply demonstrates the fact that the CR we used is very restrictive as it refuses $7 \%$ of the single replies. Due to this high sensitivity, this receiver has an overall bad performance for a mixture of replies. Because we use it after the separation step, it reduces also the success rate of the full procedure (S.+CR). For a single reply, the success rate is almost the same for $\mathrm{CR}$ and S.+CR, which is normal since the separation step cannot help for a single reply (beside from noise reduction). We note that for several replies the success rate of the full procedure is larger by $27 \%$ than the conventional receiver alone. On the overall, the full procedure has a $15 \%$ success improvement over CR.

\begin{tabular}{|l|c|c|}
\hline Case & C.R. & S.+C.R. \\
\hline \hline Single reply & 0.00 & 0.00 \\
\hline 2 replies & 0.50 & 0.58 \\
\hline 3 replies, & 0.68 & 1.24 \\
\hline 4 replies, & 0.85 & 2.30 \\
\hline More replies & 1.25 & 2.38 \\
\hline
\end{tabular}

Table 2. Recovery failure: Average number of replies decoded for failed cases for the Conventional Receiver, and for the EPA followed by the Conventional Receiver.

The improvement done by the separation step is also shown in the table 2, which present the average number of replies decoded for the failed cases. We note that the effect is not sensible for 2 replies, but increase in a sensible manner for more replies.

\section{CONCLUSION AND PERSPECTIVES}

In this paper, we have presented a practical solution to the cumbersome problem of separing any number of overlapping Mode $\mathrm{S}$ and Mode $\mathrm{A} / \mathrm{C}$ replies. This proposed solution works satisfactorily in most of the cases with a limited cost in computation (and therefore in hardware) with respect to competing solutions.

\section{ACKNOWLEDGMENT}

The authors wish to thank Selex-Sistemi Integrati and Finmeccanica Group for supporting research activities by Tor Vergata University in the area of SSR. The contribution by Delft Technical University (IRCTR and CAS) to the acquisition of real SSR signals is also gratefully acknowledged.

\section{REFERENCES}

[1] P. Bezousek, "A passive radar surveillance system VERA for ATC," in IRS'98, Munich, Germany, 1998.

[2] Gaspare Galati, "USA Patent 6,819,282 B1," 16 Nov. 2004, EU patent 02728019-7-2220-IT 0200206.

[3] N. Petrochilos and A.J. van der Veen, "Algorithms to separe overlapping secondary surveillance radar replies," in Proc. of ICASSP 2004, 17-21 May 2004, pp. II.49-53.

[4] N. Petrochilos, G. Galati, L. Mené, and E. Piracci, "Separation of multiple secondary surveillance radar sources in a real environment by a novel projection algorithm," in Proc. of ISSPIT 2005, 17-21 December 2005.

[5] Pierre Comon, "Independent component analysis, a new concept ?," Signal Processing, Special issue on HigherOrder Statistics, vol. 36, no. 3, pp. 287-314, April 1994.

[6] R. Roy and T. Kailath, "ESPRIT estimation of signal parameters via rotational invariance techniques," IEEE Trans. on acoustics, speech, and Signal Processing, vol. 37, no. 7, pp. 984-995, July 1989.

[7] A.J. van der Veen and J. Tol, "Separation of zero/constant modulus signals," in Proc. IEEE ICASSP, Munich (FRG), April 1997, pp. 3445-3448.

[8] M.C. Stevens, Secondary Surveillance Radar, Artech house, Norwood, MA, 1988.

[9] R.M. Trim, "Mode S: an introduction and overview," Electronics \& Communication Engineering Journal, vol. 2, pp. 53-59, Apr. 1990.

[10] N. Petrochilos and P. Comon, "Separation de signaux ZCM: application en radar SSR," in Proc. of GRETSI 2003, Paris, France, 8-11 September 2003.

[11] N. Petrochilos and P. Comon, "A zero-cumulant random variable and its applications," Signal Processing Magazine, 2006, Accepted.

[12] S. Haykins and all, Radar Array Processing, SpringerVerlag, 1993.

[13] R. A. Horn and C. R. Johnson, Matrix Analysis, Cambridge University Press, 1985.

[14] Luca Menè, "Acquisition and analysis of secondary surveillance radar replies," Tesi di laurea, Università di Roma Tor Vergata, Roma, Italy, 2003.

[15] Gaspare Galati and Emilio Piracci Maurizio Gasbarra, “Decoding techniques for SSR Mode S signals in high traffic environment," in Proceeding of EuMA, EURAD 05, Paris, France, 6-7 Oct. 2005. 